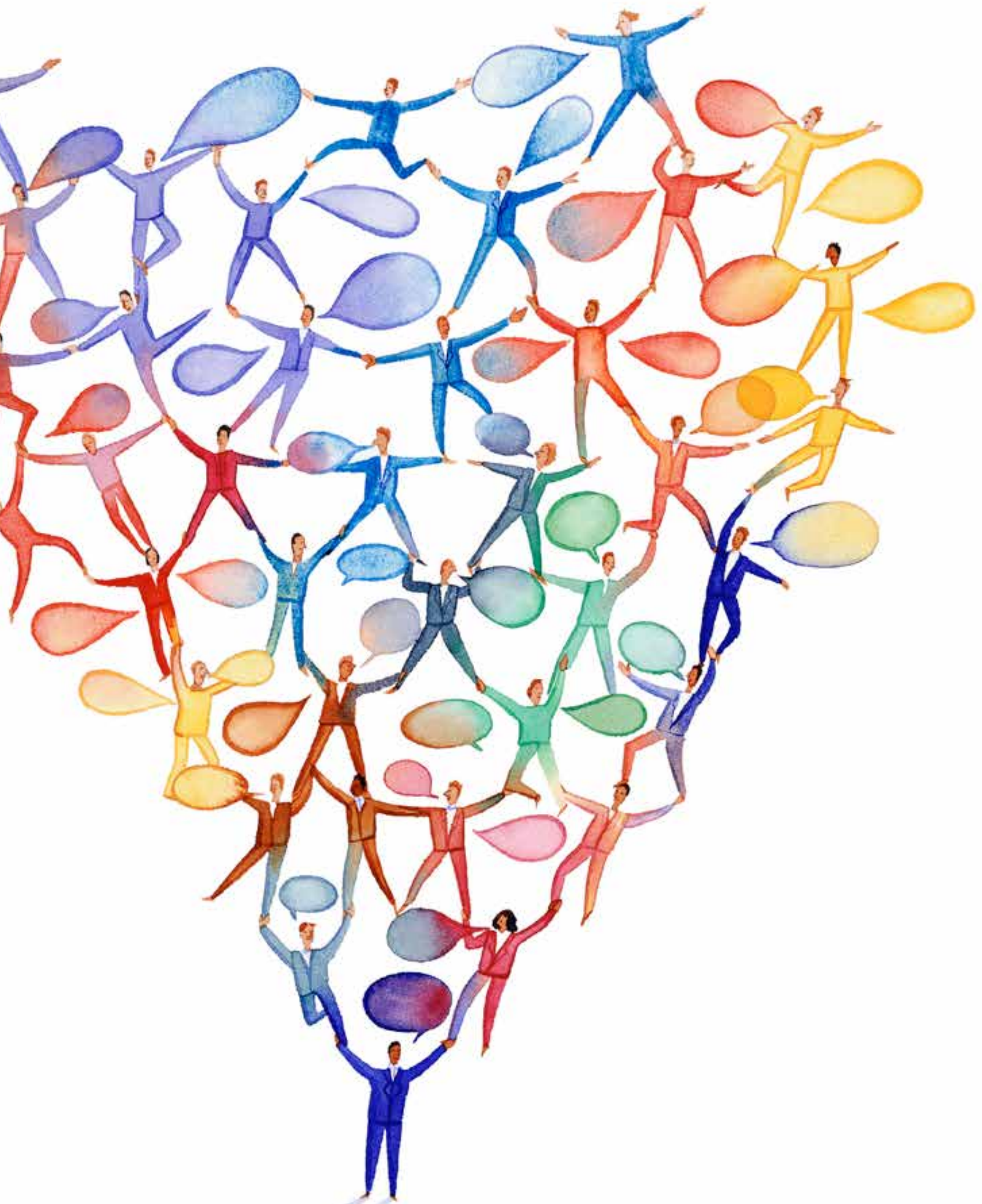

B. Tec. Senac, Rio de Janeiro, v. 42 n.1, p. 30-47, jan./abr. 2016. 
* Mestre em Ensino na Saúde pelo Programa de Pós-graduação em Ensino na Saúde da Universidade Federal de Goiás (UFG). Professora Assistente no Curso de Enfermagem da UFG Regional Jataí.

E-mail:

Igregomaia@yahoo.com.br

** Doutora em Ciências da Saúde pela Universidade de Brasília (UNB). Professora Adjunta da Faculdade de Nutrição da UFG.

E-mail: lumasa@ufg.br

*** Doutor em Ciências pela Escola de Enfermagem de Ribeirão Preto. Professor Adjunto II da UFG e Orientador do Programa de Pós-graduação em Ensino na Saúde.

E-mail:

enferluiz@yahoo.com.br

****Doutora em Ciências pela Universidade de São Paulo. Professora Associada da Faculdade de Nutrição da UFG e Orientadora do Programa de Pós-graduação em Ensino na Saúde.

E-mail:

idahelenamenezes@gmail.com

Recebido para publicação em: 24.1.2016

Aprovado em: 14.4.2016

\section{ATIVIDADES EDUCATIVAS NA SAÚDE NA PERSPECTIVA DA EDUCAÇÃO PERMANENTE EM UM MUNICÍPIO DE GOIÁS' 1}

\author{
HEALTH EDUCATIONAL ACTIVITIES IN THE \\ PERSPECTIVE OF PERMANENT EDUCATION \\ IN A CITY OF GOIÁS \\ Ludmila Grego Maia* \\ Lucilene Maria de Sousa** \\ Luiz Almeida da Silva*** \\ Ida Helena Carvalho Francescantonio Menezes****
}

\section{Resumo}

Objetivou-se analisar as atividades educativas na rede municipal de saúde na perspectiva da Política Nacional de Educação Permanente em Saúde. Estudo retrospectivo, descritivo, documental, realizado em um município do Sudoeste Goiano. Foram analisadas 82 atividades educativas, ocorridas no ano de 2012, das quais $80,5 \%$ se deram com ênfase na educação na saúde voltada à comunidade. Houve predominância das metodologias tradicionais de ensino em $67,6 \%$ e a origem das atividades educativas se deu na maior parte por demandas internas ao serviço, 86,6\%. Observa-se que, embora haja esforços contínuos para mudanças, implementar a política é um desafio, devido à sua complexidade político-pedagógica no contexto do Sistema Único de Saúde.

Palavras-chave: Educação Permanente em Saúde. Recursos Humanos em Saúde. Sistema Único de Saúde.

\section{Abstract}

The purpose was to analyze the educational activities in the municipal health network in the perspective of the National Permanent Education Policy in Health. A retrospective, descriptive and documentary study was developed in a city in the Southwest of Goiás. 82 educational activities were analyzed, held in 2012, from which $80.5 \%$ were developed with emphasis on communityoriented education in health. There was a predominance of traditional teaching methodologies in $67.6 \%$, and the educational 
activities origin was mostly by the service internal demands, in $86.5 \%$. It is noticed that, although there are continuous efforts for changes, implementing a policy is a challenge, due to its political-pedagogical complexity in the context of the Unified Health System.

Keywords: Permanent Education in Health. Human Resources in Health. Unified Health System.

\section{Resumen}

El objetivo fue analizar las actividades educativas en la red municipal de salud en la perspectiva de la Política Nacional de Educación Permanente en Salud. Un estudio retrospectivo, descriptivo, documental, realizado en una municipalidad del Sudoeste de Goiás. Se analizaron 82 actividades educativas, que se produjeron en el año 2012, de las cuales el 80,5\% ocurrió con énfasis en la educación para la salud orientada a la comunidad. Hubo un predominio de las metodologías tradicionales de enseñanza en el 67,6\% y el origen de actividades educativas ocurrió en su mayoría por demandas internas al servicio, en un $86,5 \%$. Se observa que, aunque existan esfuerzos continuados para los cambios, implementar la política es un reto, debido a su complejidad político-pedagógica en el contexto del Sistema Unificado de Salud.

Palabras clave: Educación Permanente en Salud. Recursos Humanos en Salud. Sistema Unificado de Salud.

\section{Introdução}

A educação permanente em saúde (EPS), instituída em 2004 pelo Ministério da Saúde, encontra-se fundamentada pela Política Nacional de Educação Permanente em Saúde (PNEPS) e normatizada pela Portaria GM/MS n. 1.996 de 2007. Visa atender aos propósitos da Constituição de 1988 para ordenar a formação de recursos humanos na área da Saúde (BRASIL, 1988, 2009).

É definida como sendo aprendizagem no trabalho, onde se almeja que haja por parte dos trabalhadores da saúde uma aprendizagem significativa de saberes que são essenciais ao cotidiano dos serviços. O objetivo primordial da EPS é transformar a prática profissional, por meio de ações pensadas e refletidas pela equipe enquanto atuante nas instituições de saúde e, como produto disso, espera-se uma assistência de qualidade, resolutiva e consoante com os princípios e diretrizes do Sistema Único de Saúde (SUS) (CECCIM, 2005).

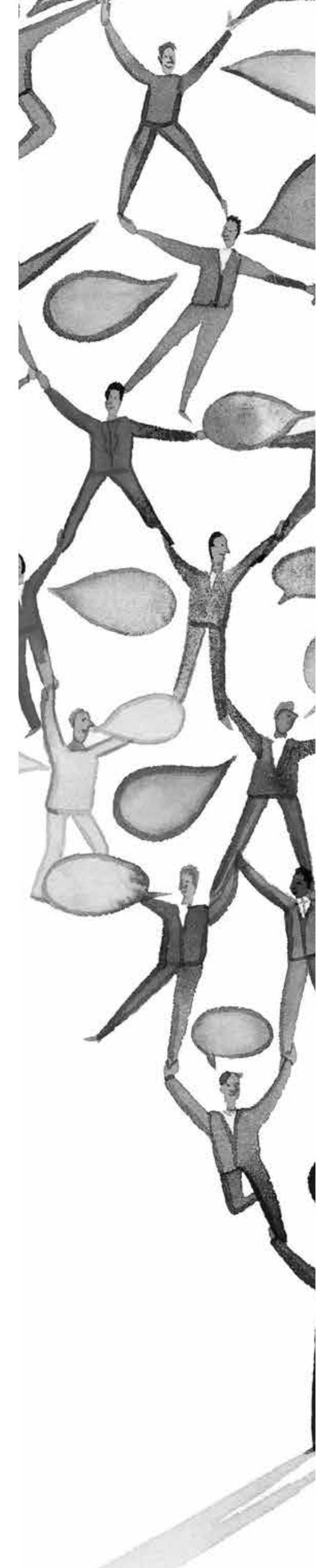


Apesar de parecer uma tarefa simples, implementar a PNEPS tem sido algo desafiador. Estudos mostram que a mesma vem sendo entendida como mudança conceitual, tomando como alicerce a educação continuada (EC), a qual é definida como processo amplo que inclui a educação formal e a informal com o objetivo de desenvolvimento pessoal e profissional dos trabalhadores de saúde (DAVIM; TORRES; SANTOS, 1999; PASCHOAL; MANTOVANI; MÉIER, 2007).

Acredita-se que, por ambos os termos coabitarem nos mesmos espaços e rodas, acabou sendo fator de confusão entre os trabalhadores da saúde, o que implica em risco para gestão, vez que aposta-se na EPS para romper paradigmas, mudar o modelo assistencial, qualificar as equipes de saúde e assim consolidar o SUS (BRASIL, 2009; CECCIM, 2005).

Alguns estudos, como Montanha; Peduzzi (2010); Peduzzi et al. (2009); Silva; Peduzzi (2011) buscaram apontar as concepções de EPS e EC por trabalhadores de saúde e demonstraram que ambas convivem no imaginário dos profissionais, sendo que a EPS, muitas vezes, é entendida como mera mudança de denominação da EC.

Tendo como premissas as recomendações da PNEPS e com atuação profissional no âmbito da atenção primária em saúde, questiona-se: As ações assumidas como educação permanente em saúde estão concernentes com a política ou reproduzem aspectos relativos a outros processos formativos, como a educação continuada?

A presente questão norteadora subsidiou o intento de verificar o atendimento às recomendações da PNEPS, em um município do Sudoeste goiano, na microrregião Sudoeste II, que teve o processo de implantação da PNEPS no ano de 2010, com a constituição da Comissão de Integração Ensino Serviço (CIES).

\section{Métodos}

Trata-se de um estudo retrospectivo, realizado por análise documental, com abordagem quantitativa. O município de estudo, localizado no Sudoeste do estado de Goiás, possui ao todo 16 unidades básicas de saúde (UBS), destas, 13 atuam dentro da Estratégia Saúde da Família (ESF), sendo três na zona rural. A cobertura estimada da ESF está em 52,4\%. Na média e alta complexidade, o município conta com três hospitais gerais, sendo um hospital sob a gestão municipal, classificado como de médio porte, com 47 leitos. Nessa mesma unidade, funcionam o Serviço de Apoio Diagnóstico e Terapêutico (SADT), o Centro de Testagem e Aconselhamento (CTA), além de ambulatório de especialidades médicas. 
Com o intuito de adequação às recomendações do Ministério da Saúde, em 2012, a Secretaria Municipal de Saúde elaborou e implementou um documento para mensuração das atividades educativas promovidas pelas unidades de saúde, com o intuito de gerenciar as atividades desenvolvidas, bem como o aprimoramento dos recursos humanos sob sua responsabilidade.

Tal documento contempla questões como período de execução, coordenação do evento, número de participantes, identificação e local da atividade, objetivo, justificativa, conteúdo programático, recursos audiovisuais utilizados, entre outros. Trata-se de um instrumento semelhante ao proposto no Plano da Ação de Trabalho e Educação, adotado pelo Ministério da Saúde na Portaria GM/MS n. 278, de 27 de fevereiro de 2014.

Para que a atividade educativa aconteça dentro do município, é adotado o seguinte fluxo: primeiro, tem-se o preenchimento desse instrumento pelo coordenador da unidade solicitante; em seguida, é enviado à coordenação de EPS da Secretaria Municipal de Saúde, que analisará a solicitação para posterior autorização e apoio logístico.

O presente estudo foi desenvolvido com base na análise desse documento disponibilizado pela Secretaria Municipal de Saúde, oriundo de todas as unidades da rede básica, do centro de testagem e aconselhamento e do núcleo de vigilância epidemiológica, que solicitaram atividades educativas da no ano de 2012.

Para proceder à análise dos documentos, considerou-se como critério de inclusão o preenchimento pelo qual deveriam constar identificação e local da atividade; justificativa; objetivos; conteúdo programático e recursos audiovisuais. Foram disponibilizados 512 documentos, os quais, depois de submetidos aos critérios de inclusão, resultaram em um total de 82 documentos a serem analisados.

Para a análise e categorização das informações, utilizou-se um instrumento que foi elaborado com base no estudo de Peduzzi et al. (2009) e na PNEPS, apresentado no Quadro 1, onde encontra-se a descrição das variáveis. 


\section{Quadro 1 - Categorização das variáveis do estudo utilizadas na análise das atividades educativas}

\begin{tabular}{|c|c|}
\hline \multirow{3}{*}{$\begin{array}{c}\text { Tipo de atividade } \\
\text { educativa }\end{array}$} & $\begin{array}{l}\text { Atividades educativas com enfoque na promoção, prevenção ou } \\
\text { recuperação da saúde. }\end{array}$ \\
\hline & Treinamentos, simpósios e seminários. \\
\hline & Reuniões gerenciais/administrativas. \\
\hline \multirow{5}{*}{ Público-alvo } & Área específica (profissionais de saúde separadamente). \\
\hline & Trabalhadores de enfermagem e médicos. \\
\hline & Todos os trabalhadores do serviço. \\
\hline & Equipes de trabalho (equipe ESF). \\
\hline & Comunidade. \\
\hline \multirow{11}{*}{$\begin{array}{l}\text { Estratégias de } \\
\text { ensino }\end{array}$} & Metodologias ativas. \\
\hline & Discussão em grupo. \\
\hline & Oficinas de trabalho. \\
\hline & Aula expositiva dialogada. \\
\hline & Aulas práticas. \\
\hline & Dinâmicas de grupo. \\
\hline & Outras. \\
\hline & Metodologia tradicional. \\
\hline & Palestras. \\
\hline & Aula expositiva. \\
\hline & Outras. \\
\hline \multirow{2}{*}{$\begin{array}{c}\text { Origem da } \\
\text { demanda pela } \\
\text { atividade educativa }\end{array}$} & Externa (solicitações de pessoas externas à equipe/comunidade). \\
\hline & Interna (emergiu dos trabalhadores). \\
\hline \multirow{3}{*}{ Duração } & $1-2$ horas \\
\hline & $2-4$ horas \\
\hline & $>4$ horas \\
\hline
\end{tabular}

Fonte: Elaborado pelos autores com base no instrumento de Peduzzi, et al. (2009) e na PNEPS (BRASIL, 2009).

Para a variável tipo de atividade educativa, foram criadas três categorias, em que a primeira listada está mais próxima da EPS, visto que compreende atividades de promoção, prevenção de doenças e recuperação da saúde, níveis de atenção à saúde que se articulam no âmbito da integralidade, que é um dos princípios do SUS e, consequentemente, da PNEPS. As duas últimas categorias foram interpretadas como mais próximas da EC.

Para a variável público-alvo, definiram-se como expressão da EPS as atividades que envolveram todos os trabalhadores do serviço ou os trabalhadores das equipes de ESF. Já as estratégias de ensino foram divididas em metodologias ativas como indicativas da EPS e metodologia tradicional voltada para EC. 
$\mathrm{Na}$ origem das demandas, consideraram-se aquelas originadas internamente ao serviço, com o potencial de terem emergido a partir da realidade das equipes de trabalho, o que positivamente aponta para a lógica da EPS. A duração das atividades foi mensurada em horas, sendo de uma a mais de quatro horas.

Os temas trabalhados nas atividades educativas foram classificados em áreas específicas, como saúde da mulher, saúde do adulto e idoso, saúde da criança e do adolescente, entre outras. Já as justificativas foram analisadas com a criação de duas categorias e seis subcategorias, compreendendo: administrativa (planejamento; qualidade da assistência; relacionamento interpessoal) e cuidado em saúde (promoção da saúde; integração equipe $x$ comunidade e prevenção de doença).

Os dados foram digitados no programa Microsoft Excel $2007^{\circledR}$ e a análise estatística descritiva foi realizada com a utilização do programa Statistical Package for Social Science ${ }^{\mathrm{TM}}$ (SPSS) versão $20.0^{\circledR}$ para Windows. Para a análise estatística dos dados, foi realizada uma caracterização das atividades encontradas, segundo as unidades de análise previamente definidas mediante o cálculo de frequências absolutas e relativas para as variáveis.

O projeto seguiu as normas para pesquisa envolvendo seres humanos, e foi aprovado pelo Comitê de Ética em Pesquisa da Universidade Federal de Goiás, n. 334.530/2013.

Há predominância de atividades educativas com enfoque na promoção, prevenção ou recuperação da saúde

\section{Resultados}

A distribuição das variáveis por tipo de atividade educativa e público-alvo, descrita na Tabela 1, mostra que há predominância de atividades educativas com enfoque na promoção, prevenção ou recuperação da saúde, com 66 $(80,5 \%)$ das atividades. Já os treinamentos, simpósios e seminários corresponderam a 15 (18,3\%). O público-alvo das atividades educativas analisadas foi a comunidade, representando 58 (70,7\%), seguida de 13 (15,8\%) para áreas específicas. 


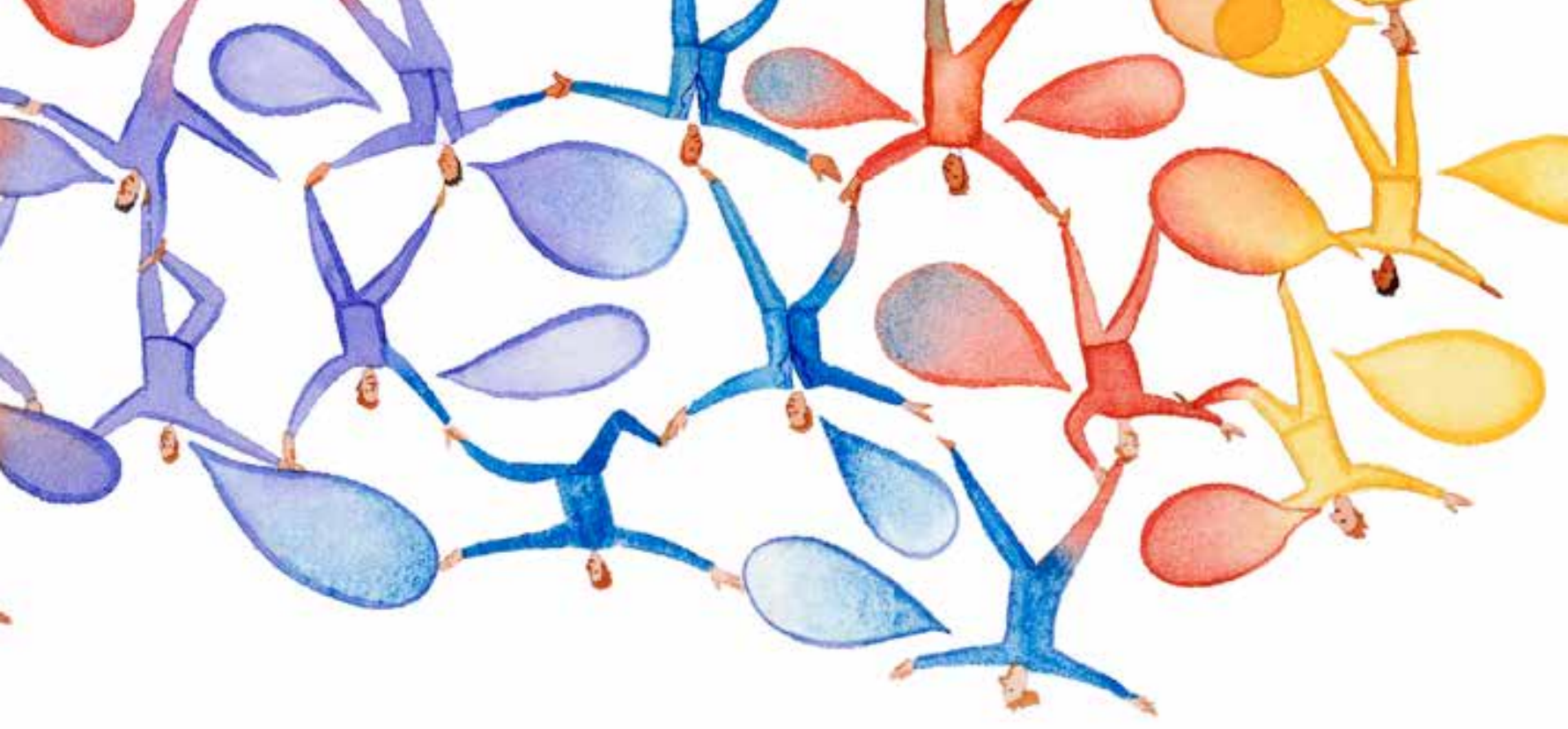

Tabela 1 - Tipos de atividades educativas e público-alvo na rede de saúde. Município do Sudoeste goiano, 2012

\begin{tabular}{|c|c|c|}
\hline $\begin{array}{c}\text { Tipo de atividade educativa } \\
\text { Atividades educativas com enfoque na promoção, prevenção ou } \\
\text { recuperação da saúde }\end{array}$ & 66 & $\mathbf{N}$ \\
\hline Treinamentos, simpósios, seminários & 15 & 18,3 \\
\hline Reuniões gerenciais/administrativas & 1 & 1,2 \\
\hline Total & $\mathbf{8 2}$ & $\mathbf{1 0 0}$ \\
\hline Público-alvo & & \\
\hline Comunidade & 58 & 70,7 \\
\hline Área específica (profissionais de saúde separadamente) & 13 & 15,8 \\
\hline Todos os trabalhadores do serviço & 7 & 8,5 \\
\hline Equipes de trabalho (equipe ESF) & 4 & 5,0 \\
\hline Total & $\mathbf{8 2}$ & $\mathbf{1 0 0}$ \\
\hline
\end{tabular}

Fonte: Elaborado pelos autores com base nos dados da pesquisa, município do Sudoeste goiano, 2012.

As metodologias ativas foram utilizadas em $25(32,4 \%)$ atividades e as tradicionais em $52(67,6 \%)$. Entre as metodologias ativas, destacaram-se as aulas práticas 13 (52\%). Já as estratégias tradicionais foram utilizadas em $52(65,8 \%)$ de todas as atividades educativas, com predominância de palestras, $25(48,1 \%)$, conforme se descreve na Tabela 2.

A variável origem da demanda mostra que as atividades educativas começaram internamente ao serviço, ou seja, em 71 (86,6\%) dos documentos analisados. No que tange à duração das atividades realizadas, dos 82 documentos, 37 (45,1\%) não informaram a carga horária da atividade e $25(55,5 \%)$ apresentaram duração entre uma a duas horas de atividade (Tabela 2). 
Tabela 2 - Distribuição das informações presentes no instrumento das atividades educativas realizadas na rede de saúde segundo as variáveis: estratégia de ensino, origem da demanda e carga horária. Município do Sudoeste goiano, 2012

\begin{tabular}{|c|c|c|}
\hline Estratégia de ensino ativa & $\mathbf{N}$ & $\%$ \\
\hline Aulas práticas & 13 & 52 \\
\hline Dinâmicas de grupo & 6 & 24 \\
\hline Aula expositiva dialogada & 3 & 12 \\
\hline Oficinas de trabalho de grupo & 3 & 12 \\
\hline Total & 25 & 100 \\
\hline \multicolumn{3}{|l|}{ Estratégia de ensino tradicional } \\
\hline Palestras & 25 & 48,1 \\
\hline Aula expositiva & 16 & 30,7 \\
\hline Outras* & 11 & 21,2 \\
\hline Total & 52 & 100 \\
\hline \multicolumn{3}{|l|}{ Origem da demanda } \\
\hline Interna (emergiu dos trabalhadores) & 71 & 86,6 \\
\hline Externa (solicitações de pessoas externas à equipe/comunidade) & 9 & 11 \\
\hline Não informado & 2 & 2,4 \\
\hline Total & 82 & 100 \\
\hline \multicolumn{3}{|l|}{ Carga horária } \\
\hline $1-2$ horas & 25 & 30,5 \\
\hline $2-4$ horas & 16 & 19,5 \\
\hline$>4$ horas & 4 & 4,9 \\
\hline Sem carga horária informada & 37 & 45,1 \\
\hline Total & 82 & 100 \\
\hline
\end{tabular}

Fonte: Elaborado pelos autores com base nos dados da pesquisa, município do Sudoeste goiano, 2012.

*Outras: leituras e discussão de documentos.

Dos 82 (100\%) documentos, somente um (1,22\%) não informou a temática trabalhada. Os principais temas solicitados foram direcionados à saúde do adulto e do idoso $(n=21 ; 26 \%)$, saúde da criança e do adolescente $(\mathrm{n}=20 ; 24 \%)$ e a questões administrativas $(\mathrm{n}=9$; 15\%), como mostra a Figura 1.

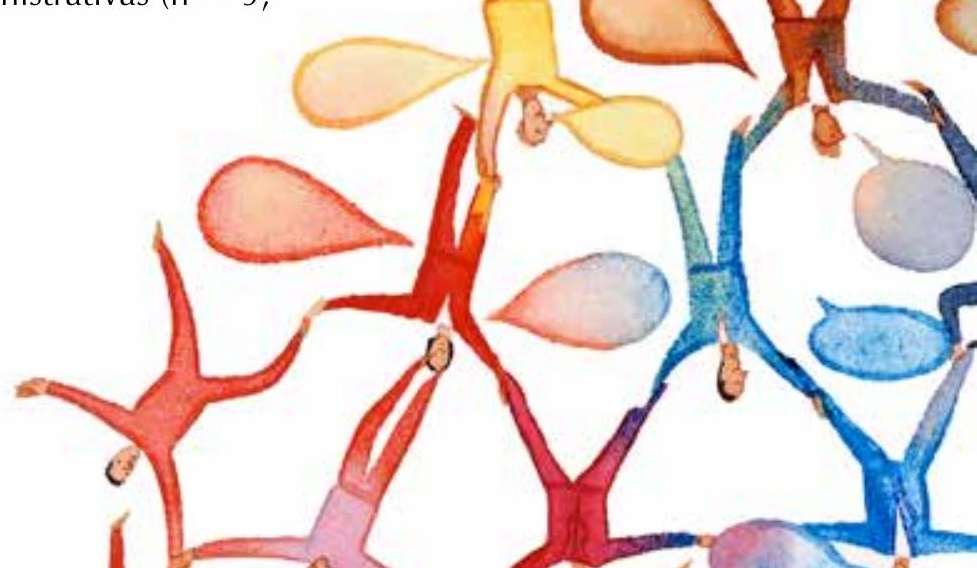


Figura 1 - Temas trabalhados nas atividades educativas na rede de saúde. Município do Sudoeste goiano, 2012

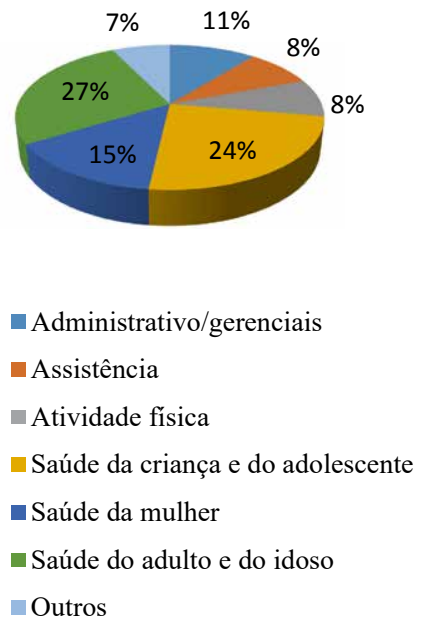

Fonte: Elaborado pelos autores com base nos dados da pesquisa, município do Sudoeste goiano, 2012.

A justificativa da ação educativa esteve presente em $74(89,1 \%)$ dos documentos analisados e foi categorizada em: administrativa, 11 (14,9\%) e cuidado em saúde, 63 (85,1\%). Dessas duas categorias, destacam-se as subcategorias planejamento - 5 (45,4\%) e promoção da saúde - $42(66,7 \%)$, respectivamente (Tabela 3 ).

Tabela 3 - Categorias e subcategorias a partir das justificativas das atividades educativas realizadas na rede de saúde. Município do Sudoeste goiano, 2012

\begin{tabular}{|c|c|c|c|}
\hline Categoria & Subcategoria & $\mathbf{N}$ & $\%$ \\
\hline \multirow{3}{*}{ Administrativa } & Planejamento & 5 & 45,4 \\
\hline & Qualidade da assistência & 4 & 36,4 \\
\hline & Relacionamento interpessoal & 2 & 18,2 \\
\hline \multicolumn{2}{|c|}{ Total } & 11 & 100 \\
\hline \multirow{3}{*}{ Cuidado em saúde } & Promoção da saúde & 42 & 66,7 \\
\hline & Integração equipe $x$ comunidade & 14 & 22,2 \\
\hline & Prevenção de doença & 7 & 11,1 \\
\hline \multicolumn{2}{|c|}{ Total } & 63 & 100 \\
\hline
\end{tabular}

Fonte: Elaborado pelos autores com base nos dados da pesquisa, município do Sudoeste goiano, 2012.

Rio de Janeiro, v. 42 n.1, p. 30-47, jan./abr. 2016. 


\section{Discussão}

No município de estudo, a prática da EPS tem se dado de forma fragmentada e pouco efetiva, como foi evidenciado pelas categorias analisadas. Existe maior proximidade de ações com participação da comunidade e poucas ações educativas voltadas para os trabalhadores.

Merhy (2015) refere que a educação permanente busca, além da produção de novos conhecimentos, novos processos de formação, ambos construídos coletivamente a partir dos processos de trabalho, porque é nesse contexto do exercício da profissão que os trabalhadores efetivamente irão se formar. Para o autor, o mundo do trabalho sempre implica em processos formativos.

A partir dessa afirmativa, é relevante discutir que as atividades educativas analisadas, denominadas como EPS, podem ter sido classificadas como tal de forma equivocada. A partir da formulação da PNEPS, esse novo conceito EPS foi inserido no cenário do SUS de maneira simplista, o que pode ser corroborado por outros estudos, mostrando que os profissionais entenderam a EPS como mudança conceitual, sem levar em consideração a sua estrutura conceitual e metodológica (FORTUNA et al., 2011; MANCIA; CABRAL; KOERICH, 2004; MARANDOLA et al., 2009).

Pelo presente estudo, as atividades educativas estiveram voltadas para comunidade e o enfoque na promoção, prevenção ou recuperação da saúde teve grande expressão, o que pode ser visto positivamente, pois leva a pensar que as equipes têm enxergado a necessidade de se articular ações educativas nesses três níveis de assistência, potencializando a possibilidade de transformar o seu processo de trabalho com vistas à construção de um modelo mais próximo da integralidade (PEDUZZI et al., 2009; SILVA; PEDUZZI, 2011).

A partir do enfoque prioritário voltado à comunidade, que é um dos eixos do quadrilátero representando o controle social, observa-se que há um olhar das equipes em direção às necessidades de saúde dos usuários, o que amplia possibilidades de respostas mais abrangentes e resolutivas para as necessidades de saúde, assim como materializa o encontro da saúde com 
a educação e a cidadania, o que significa controle social, práticas participativas, alteridade com os movimentos populares e ligações com a sociedade civil (BRASIL, 2009; CECCIM; FERLA, 2008).

A segunda categoria predominante (treinamentos, simpósios e seminários) caracteriza-se por serem atividades próximas à EC, visto seu caráter fragmentado e pontual, quando da análise dos objetivos e justificativas na proposição das atividades. A EC parte da percepção da necessidade individual de aprimoramento profissional, quando da sua atuação no campo de trabalho, tendo um papel de preencher lacunas na sua formação, objetivando qualificar o trabalhador para melhor exercer a sua função (DAVIM; TORRES; SANTOS, 1999; MONTANHA; PEDUZZI, 2010).

Para o quadrilátero da formação, esse eixo constitui o trabalho e os serviços de saúde. A literatura evidencia que, para a transformação das práticas profissionais e do processo de trabalho, a proposta da EPS é um recurso estratégico. No entanto, há a necessidade da participação da equipe multiprofissional, evitando-se a fragmentação disciplinar nas ações de educação na saúde, só assim essas mudanças podem ser mais efetivas (BRASIL, 2009; CECCIM, FEUERWERKER, 2004).

Para Ceccim (2005) ou se constituem equipes multiprofissionais, coletivos de trabalho, "ou colocamos em risco a qualidade de nosso trabalho, porque sempre seremos poucos, sempre estaremos desatualizados, nunca dominaremos tudo o que se requer em situações complexas de necessidades em direitos à saúde" (CECCIM, 2005, p. 163).

Outro aspecto relevante e prioritário da EPS se refere às metodologias de ensino. A EPS dá ênfase à adoção de práticas pedagógicas emancipatórias pelos serviços de saúde, para que provoquem mudanças significativas no processo de trabalho das equipes de saúde (BRASIL, 2009).

Neste estudo, observou-se que há uma fragilidade quanto ao uso dessas metodologias e que ainda predominam as metodologias tradicionais de ensino. Alves (2007) aponta que, apesar da emergência de um novo discurso no campo da educação em saúde, ainda prevalecem práticas educativas hegemônicas. No enfoque da EPS, é esperado que haja uma mudança significativa na concepção e nas práticas de capacitação dos trabalhadores dos serviços, modificando as estratégias educativas a partir da prática como fonte de conhecimento e de problemas, problematizando o próprio fazer (BRASIL, 2009).

Estudos apontam que para os trabalhadores o principal fator que prejudica as capacitações é a utilização de metodologias inadequadas. As escolhas metodológicas nas capacitações são fundamentais para a compreensão de 
seus resultados e o impacto das capacitações depende do envolvimento e da satisfação do público-alvo e isso está intimamente ligado ao método empregado no seu desenvolvimento (LIMA et al., 2015; SILVA; OGATA; MACHADO, 2007).

Em um estudo recente, Lima et al (2015) referem que o uso de metodologias ativas possibilita um maior engajamento dos profissionais de saúde e o confronto da realidade dos serviços com a vivência de metodologias inovadoras. Isso é fator positivo para promoção de mudanças nas práticas educacionais, a partir de vivências do processo de aprendizagem em uma perspectiva construtivista, sendo essa a proposta adotada pela PNEPS.

Outro fator a ser considerado para EPS é o tempo que se dispende para realizar determinadas ações educativas. Apesar do percentual elevado de horas não informadas, percebe-se que a maioria das atividades compreende o período de uma a duas horas de duração. Outros estudos que quantificaram as atividades em termos de horas apontam que a longa duração das atividades pode ser fator prejudicial no que tange à adesão dos trabalhadores. No entanto, não há consenso na literatura quanto ao tempo mínimo ou máximo preconizado. Cabe ressaltar também que para a aprendizagem ser significativa e provocar mudanças no cotidiano das organizações, atividades de curta duração podem não permitir a imersão do profissional para um processo de (des)construção e (re)construção a partir de suas vivências na realidade dos serviços de saúde (SILVA; OGATA; MACHADO, 2007; STROSCHEIN; ZOCCHE, 2011).

Quanto à temática trabalhada nas atividades, foi visto que os temas foram classificados em áreas específicas de atuação da atenção básica, mostrando que houve predominância das atividades com foco na saúde do adulto e do idoso, seguida de atividades voltadas para saúde da criança e do adolescente. Outros estudos, como Viana et al. (2008) e Cardoso (2012), mostram que os conteúdos trabalhados estão sempre relacionados às áreas temáticas que acompanham os grandes ciclos vitais (hipertensos, diabéticos, criança, mulher, idosos), o que vai ao encontro dos dados do presente, uma vez que os mesmos não apresentam uma visão preventiva, estando mais no âmbito pós acometimento de danos.

Esse achado justifica-se pelo fato de as ações programáticas já estabelecidas pelo Ministério da Saúde serem trabalhadas de forma rotineira nas unidades de saúde, tendo programas e agendas pré-estabelecidos já incorporados pela comunidade. Tal perspectiva mostra que, embora a PNEPS esteja despendendo esforços para mudança de foco na atenção em saúde, passando de modelo biomédico para o modelo preventivo, os profissionais ainda tendem a esperar que os agravos aconteçam para, posteriormente, educar a população.

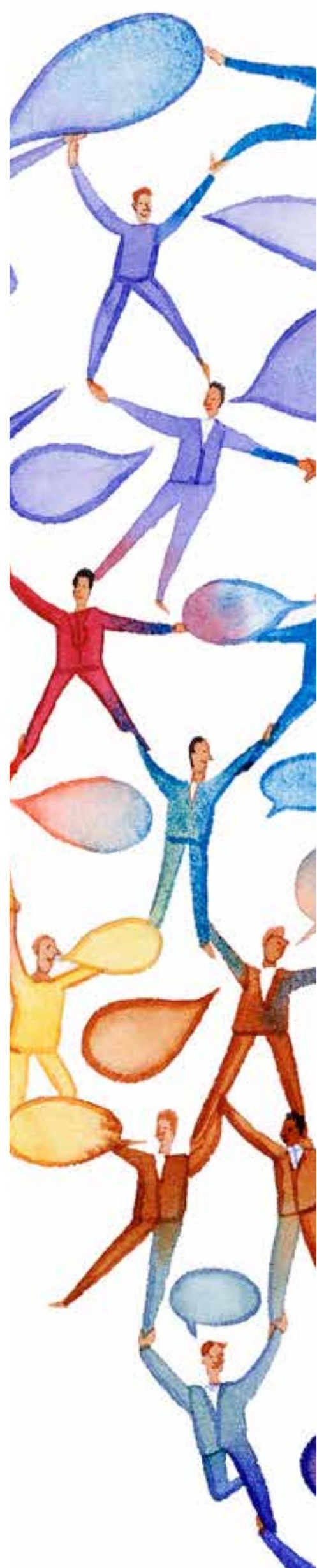


pondam às necessidades da comunidade e, quando o processo de trabalho está comprometido com a EPS, impulsiona os trabalhadores de saúde a produzir uma nova maneira de cuidar, modificando então a sua prática (BRASIL, 2009; MERHY, 2005; PAULINO et al. 2012).

Embora o estudo tenha ocorrido na maioria das unidades de saúde, o que permitiu pela primeira vez conhecer o perfil das atividades educativas na saúde desenvolvidas no município, foi analisado um número reduzido de solicitações de treinamento em função do preenchimento incompleto do formulário.

\section{Considerações finais}

Os resultados deste estudo sugerem que ainda é pouco consistente a compreensão no município sobre a PNEPS. Percebe-se que as ações de educação na saúde que ocorreram no município, apesar de nomeadas como EPS, ainda possuem aspectos ligados a EC, principalmente ao se observar as metodologias adotadas pelas equipes nas ações de educação executadas.

Um dos pontos a ser discutido é o grande número de documentos apresentados, que não responderam ao instrumento padronizado. Questiona-se a importância do preenchimento dessa solicitação de treinamento para os profissionais. Será que a mesma contempla as necessidades e os anseios dos profissionais? Ou foi entendida como instrumento meramente burocrático para o registro de atividades? Esse foi um dos fatores limitantes do estudo, tendo em vista o número reduzido de atividades que puderam ser avaliadas.

A complexidade político-pedagógico da PNEPS precisa ser melhor entendida para que as equipes se apropriem da EPS como importante ferramenta para transformação do processo de trabalho e transformação da realidade local.

Os resultados dessa pesquisa permitiram que algumas condutas fossem adotas no intuito de (re)significar a EPS. Assim, foram desenvolvidas algumas ações exitosas, como: promoção de um curso para os profissionais de saúde sobre a EPS junto à CIES Regional Sudoeste II; reuniões de apoio para implantação do núcleo de EPS no município e, por fim, foi incluída uma disciplina de núcleo livre sobre a temática na grade curricular do curso de Enfermagem da instituição formadora da região, UFG, possibilitando que haja uma aproximação dos acadêmicos com a EPS.

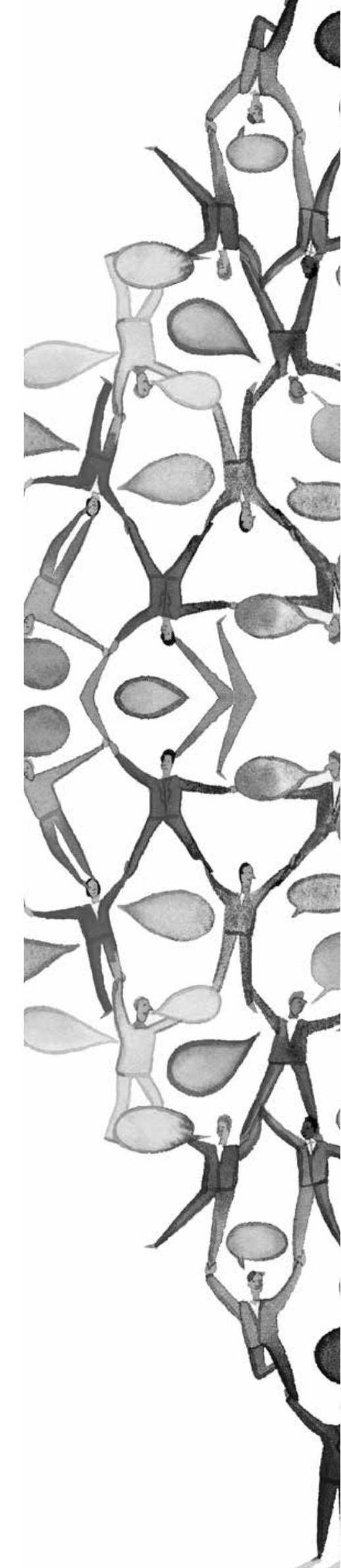

B. Tec. Senac, Rio de Janeiro, v. 42 n.1, p. 30-47, jan./abr. 2016. 

scielo.php?script=sci_arttext\&pid=S0104-11692011000200025\&lng=pt\&nrm =iso > . Acesso em:18 jan. 2014.

FUNDAÇÃO NACIONAL DE SAÚDE (Brasil). Diretrizes de educação em saúde visando à promoção da saúde: documento base-documento I. Brasília, DF, 2007. p.70.

LIMA, V. V. et al. Ativadores de processos de mudança: uma proposta orientada à transformação das práticas educacionais e da formação de profissionais de saúde. Ciência \& Saúde Coletiva, Rio de Janeiro, v. 20, n. 1, p. 279-288, jan. 2015. Disponivel em <http://www.scielo.br/scielo.php?script=sci_arttext\&pid=S1413$81232015000100279 \&$ Ing=en\&nrm=iso >. Acesso em: 21 jun 2015.

MACHADO, M. F. A. S. et al. Integralidade, formação de saúde, educação em saúde e as propostas do SUS: uma revisão conceitual. Ciência e Saúde Coletiva, Rio de Janeiro, v. 12, n. 2, abr. 2007. Disponível em: <http://www.scielo.br/scielo.php?script=sci_ arttext\&pid=S1413-81232007000200009\&lng=en\&nrm = iso $>$. Acesso em: 18 jan. 2014.

MANCIA, J. R.; CABRAL, L. C.; KOERICH, M. S. Educação permanente no contexto da enfermagem e na saúde. Revista Brasileira de Enfermagem, Brasília, DF, v. 57, n. 5, p.605-610, set./out. 2004.

MARANDOLA, T. R. et al. Educação permanente em saúde: conhecer para compreender. Revista Espaço para a Saúde, Londrina, v. 10, n. 2, p. 53-60, jun. 2009.

MERHY, E. E. O desafio que a educação permanente tem em si: a pedagogia da implicação. Interface: comunicação, saúde, educação, Botucatu, v. 9, n. 16, p. 161-177, set. 2004/fev. 2005.

MERHY, E. E. Educação permanente em movimento: uma política de reconhecimento e cooperação, ativando os encontros do cotidiano no mundo do trabalho em saúde, questões para os gestores, trabalhadores e quem mais quiser se ver nisso: artigo de opinião. Saúde em Redes, v. 1 n. 1, p. 7-14, 2015.

MONTANHA, D.; PEDUZZI, M. Educação permanente em enfermagem: levantamento de necessidades e resultados esperados segundo a concepção dos trabalhadores. Revista da Escola de Enfermagem da USP, São Paulo, v. 44, n. 3, set. 2010.

PASCHOAL, A. S.; MANTOVANI, M. F.; MEIER, M. J. Percepção da educação permanente, continuada e em serviço para enfermeiros de um hospital de ensino. Revista da Escola de Enfermagem da USP, São Paulo, v. 41, n. 3, set. 2007. Disponível em: <http://www.scielo.br/scielo.php?script=sci_arttext\&pid=\$0080-62342007000 300019\&lng=en\&nrm=iso >. Acesso em: 6 maio 2013.

PAULINO, V. C. P. et al. Ações de educação permanente no contexto da estratégia saúde da família. Revista de enfermagem da UERJ, Rio de Janeiro, v. 20, n.3, p.368-373, jul./set. 2012.

PEDUZZI, M. et al. Atividades educativas de trabalhadores na atenção primária: concepções de educação permanente e de educação continuada em saúde presentes 
no cotidiano de Unidades Básicas de Saúde em São Paulo. Interface: comunicação, saúde, educação, Botucatu, Botucatu, v. 13, n. 30, p.121-134, set. 2009.

SILVA, J. A. M.; OGATA, M. N.; MACHADO, M. L. T. Capacitação dos trabalhadores de saúde na atenção básica: impactos e perspectivas. Revista Eletrônica de Enfermagem, v. 9, n. 2, p. 389-401, 2007.

SILVA, J. A. M.; PEDUZZI, M. Educação no trabalho na atenção primária à saúde: interfaces entre a educação permanente em saúde e o agir comunicativo. Saúde e Sociedade, São Paulo, v. 20, n. 4, dez. 2011. Disponível em: <http://www.scielo.br/ scielo.php?script=sci_arttext\&pid=S0104-12902011000400018 \&lng=pt\&nrm=iso>. Acesso em: 18 jan. 2014.

STROSCHEIN, K. A.; ZOCCHE, D. A. A. Educação permanente nos serviços de saúde: um estudo sobre as experiências realizadas no Brasil. Trabalho, Educação, Saúde [online], Rio de Janeiro, v. 9, n. 3, p. 505-519, nov. 2011. Disponível em: <http://www. scielo.br/scielo.php?script=sci_arttext\&pid=S1981-77462011000300009 \&lng=pt\&nrm=iso >. Acesso em: 21 abr. 2013.

TRONCHIN, D. M. R. et al. Educação permanente de profissionais de saúde em instituições públicas hospitalares. Revista da Escola de Enfermagem USP, São Paulo, v. 43, n. 2, dez. 2009. Disponível em: <http://www.scielo.br/scielo. php?script=sci_arttext\&pid=S008062342009000600011\&lng=pt\&nrm=iso $>$. Acesso em: 18 jan. 2014.

VIANA, A. S. et al. (Org.). Recursos humanos na atenção básica, estratégias de qualificação e pólos de educação permanente no Estado de São Paulo. São Paulo: Centro de Estudos de Cultura Contemporânea/Consórcio Medicina USP, 2008. (Cadernos de atenção básica: estudos avaliativos, 4).
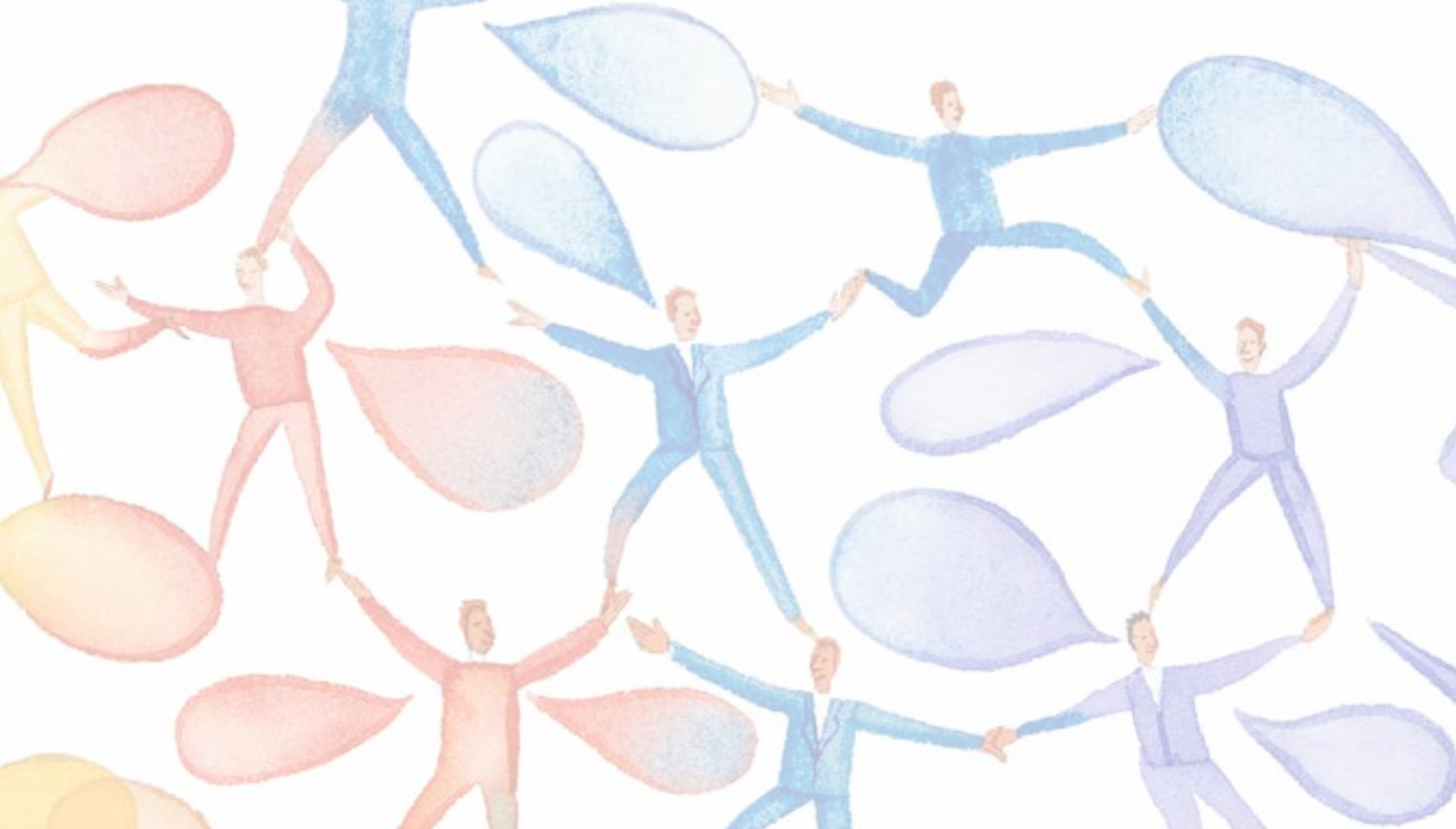

B. Tec. Senac, Rio de Janeiro, v. 42 n.1, p. 30-47, jan./abr. 2016. 